\title{
Combination and Compression of Multiple Optical Pulses in Nonlinear Fibers with the Exponentially Decreasing Dispersion
}

\author{
Qian Li, Ziyun Jian, Wei Lu, K. Nakkeeran, K. Senthilnathan, and P. K. A. Wai
}

\begin{abstract}
We propose a simple scheme to generate high energy ultrashort pulses by combination and compression of multiple input pulses which share the same chirp profile. First, the multiple raised-cosine pulses in the input pulse train are modulated by a phase modulator in which each modulation cycle covers two, three, four, or five pulses. Then, the modulated pulses are launched into a nonlinear fiber with the exponentially decreasing dispersion. We find that these pulses initially coalesce into a single pulse whose pulse profile is nearly hyperbolic secant, which then undergoes self-similar compression. Thus in the proposed method, first the combination of the multiple optical pulses occurs and then self-similar compression takes over. Besides, we also report the generation of ultrashort pulses by combination and compression of multiple hyperbolic secant pulses with the same chirp. The numerical results reveal that the resulting ultrashort pulse possesses a large portion of the input pulses for both raised-cosine and hyperbolic secant pulses. However, the compression factor and energy ratio are relatively higher for the hyperbolic secant pulses when compared to the raised-cosine pulses.
\end{abstract}

Index Terms-Computational modeling, fibers, pulse compression, nonlinear optics.

\section{INTRODUCTION}

$\mathrm{T}^{\mathrm{N}}$ recent years, the generation of high energy ultrashort $\mathbf{I}_{\text {pulses (USPs) is of great interest as these USPs find a wide }}$ range of applications in ophthalmology, nonlinear microscopy, micro-machining, and ultrahigh bit rate communication system [1-3]. It is already reported that the high energy USPs could be generated from the fiber lasers [4] and fiber amplifiers [5, 6]. However, these fiber lasers are typically complex and also they are not economically viable. The maximum output power from a fiber laser is limited by the thermal tolerance and the nonlinear effects, namely, stimulated Raman scattering (SRC) and stimulated Brillouin scattering (SBS) [7]. Instead of achieving USPs from complicated laser systems, pulse compression can be an alternative way. Pulse compression techniques including adiabatic pulse compression [8] and higher-order soliton compression [9] have been proposed for generating the USPs. In the adiabatic pulse compression, it is challenging to satisfy the adiabatic condition which typically demands a monotonically decreasing dispersion profile along the propagation direction for a long fiber length [10]. Further, the maximum compression factor is typically limited to $\sim 20$ [11]. The higher-order soliton compression helps achieve a high degree of compression in a short fiber length, but at the cost of significant pedestal generation which, in general, leads to nonlinear interactions between neighboring solitons. For example, compression of a 15-th order soliton can achieve a compression factor of 60 , but up to $80 \%$ of the compressed pulse energy appears as pedestal [9]. Further, the compression of a higher order soliton has been studied in dispersion decreasing fibers with a compression factor of 55 and a pedestal energy of $28 \%$ [11]. Thus, the conventional pulse compression techniques do not provide the expected and desired USPs. Thus an effective and compact compressor capable of generating high quality pulses with large compression factor is needed.

Moores pointed out the existence of an exact chirped soliton in nonlinear optical fibers with either constant or exponentially varying dispersion in 1996 [12]. It has also been suggested that these chirped solitary waves could be compressed more efficiently if the dispersion decreases approximately exponentially [12]. Since then, the self-similar pulse compression technique has attracted much attention. One of the salient features of self-similar compression is that it facilitates the rapid compression without satisfying the adiabatic condition [13]. Kruglov et al employed the self-similar analysis to obtain the linearly chirped solitary wave pulses and discussed efficient pulse compression in an optical fiber where both the dispersion and nonlinearity vary exponentially $[14,15]$. An efficient and compact pulse compressor, capable of producing nearly chirp-free and pedestal-free USPs, has been modeled using non-uniform fiber Bragg gratings with exponentially decreasing dispersion [16, 17]. Further, the generation of self-similar USPs at $850 \mathrm{~nm}$ has also been investigated in a tapered photonic crystal fiber (PCF) [18]. Recently, multiple pulses have been used to generate high-repetition-rate pulse train [19-23]. Olupitan et al. studied the possibility of generating a train of USPs by injecting multiple $\mathrm{RC}$ or hyperbolic secant optical pulses into a solid core PCF as well as chloroform-filled PCF [20]. Besides, Olupitan et al. also studied the robustness of such a compression scheme by perturbing the loss coefficient of the solid core PCF as well as the chloroform-filled PCF [21]. The presence of amplitude and frequency modulation of the seed wave have significant impact on the formation of the pulse train [22]. In addition, the generation of a train of USPs with high-repetition-rate has been reported based on the beating of a dual-frequency optical signal [23]. Contrary to the multiple input pulses of separate initial linear chirp across each pulse [19-23] in the generation of high-repetition-rate pulse train, in this paper, we address the issue of what would happen to the pulse train if the multiple input pulses share the same linear 
chirp across the entire pulse train. Our initial results with combination and compression of multiple optical pulses have been reported in $[24,25]$. As a first step, input pulse train is modulated by a phase modulator in which the modulation cycle covers two, three, four or five pulses. In other words, one could also assume that a number of low repetitive rate pulses of the same wavelength is multiplexed. The multiplexed beam is phased modulated by a phase modulator to achieve the same chirp profile across the multiple pulses. As a next step, the modulated pulses which have the same linear chirp are injected into a nonlinear optical fiber with exponentially decreasing dispersion. These multiple input pulses first coalesce into a single pulse and then the resulting combined single pulse undergoes compression continuously. We find that the compressed pulse attains a hyperbolic secant profile with a large portion of the input pulses' energy. Thus, the energy of final compressed pulse is nearly thrice when compared to that of the single pulse in the initial RC pulse train. We also study the formation of high energy USPs with multiple hyperbolic secant input pulses. The proposed pulse compression scheme is simple and can provide the high energy USPs within a short fiber length.

The paper is organized as follows. Section II introduces the theoretical model for describing the pulse propagation in dispersion varying nonlinear optical fibers. Section III discusses the formation of high energy USPs, via combination and compression, using multiple chirped RC pulses in a nonlinear optical fiber with exponentially decreasing dispersion. In Section IV, we show the compression of multiple chirped hyperbolic secant pulses and different pulse-to-pulse separation. Besides, we also study the combination length for both $\mathrm{RC}$ and hyperbolic secant pulse profiles with different initial pulse number and pulse width. In Section V, we address the role of initial chirp on the multiple pulse compression and study the pulse compression performance if the initial pulse chirp or dispersion decay rate varies. In Section VI, we summarize the research findings.

\section{THEORETICAL MODEL}

Pulse evolution in a dispersion varying nonlinear optical fiber is governed by the generalized nonlinear Schrödinger equation (GNLSE) [26].

$$
\begin{aligned}
& \frac{\partial A}{\partial z}+\frac{i \beta_{2}(z)}{2} \frac{\partial^{2} A}{\partial t^{2}}= \\
& \quad i\left(\gamma\left(\omega_{0}\right)+i \gamma_{1} \frac{\partial}{\partial t}\right)\left(A(z, t) \int_{0}^{\infty} R\left(t^{\prime}\right)\left|A\left(z, t-t^{\prime}\right)\right|^{2} d t^{\prime}\right),
\end{aligned}
$$

where the parameters $A, z$ and $t$ represent the amplitude of the slowly varying pulse envelope, distance and time, respectively. $\beta_{2}(z)$ and $\gamma$ are the second-order dispersion and nonlinearity coefficient of the fiber, respectively. Here $\beta_{2}(z)$ is assumed to decrease exponentially as $\beta_{2}(z)=\beta_{20} \exp (-\sigma z)$, where $\beta_{20}$ is the initial fiber dispersion and $\sigma$ is the decay rate. In this study, $\gamma$ is assumed to be a constant along the fiber length. $\gamma_{1} \approx \gamma / \omega_{0}$, where $\omega_{0}$ is the center frequency and is chosen to be 1550 $\mathrm{nm}$. The nonlinear response function is given by $R(t)=\left(1-f_{R}\right) \delta(t)+f_{R} h_{R}(t)$, where $f_{R}=0.18$, and the Raman response function $h_{R}$ is determined from the experimental fused silica Raman cross-section [27].

\section{CHIRPED RC PULSES}

\section{A. Input Pulse Shape}

In this section, we consider the compression of multiple chirped RC pulses in the form

$$
\begin{aligned}
& \frac{\sqrt{P_{0}}}{2}\left(1+\cos \left(\pi t / T_{0}\right)\right) \exp \left(i \alpha_{20} t^{2} / 2\right) \\
& t / T_{0} \in[-N, N] \quad(N \text { is odd })
\end{aligned}
$$

or

$$
\begin{aligned}
& \frac{\sqrt{P_{0}}}{2}\left\{1+\cos \left[\pi\left(t / T_{0}+1\right)\right]\right\} \exp \left(i \alpha_{20} t^{2} / 2\right) \\
& t / T_{0} \in[-N, N] \quad(N \text { is even })
\end{aligned}
$$

Here $N$ is the number of pulses sharing part of the same chirp profile. The parameters $\alpha_{20}, P_{0}$, and $T_{0}$ are the initial chirp, peak power and pulse width, respectively. The raised cosine pulse train may be generated from beating of two frequency optical signals, and its pulse-to-pulse separation is fixed and equals to twice of initial pulse width $T_{0}$. In this investigation, the fiber is chosen to have an exponentially decreasing second-order dispersion and the nonlinearity remains a constant. The decay rate of the second-order dispersion is related to the initial chirp and dispersion coefficient as $\sigma=\alpha_{20} \beta_{20}$. The fiber parameters considered are $\beta_{20}=-200 \mathrm{ps}^{2} / \mathrm{km}, \sigma=53 / \mathrm{km}, \gamma=20 / \mathrm{W} / \mathrm{km}$ and the fiber length $L=80 \mathrm{~m}$. In what follows, we delineate the compression of multiple chirped RC pulses with various numbers in the input.

\section{B. Two Chirped RC Pulses}

First, we consider the compression of two chirped RC pulses in a nonlinear fiber with exponentially decreasing dispersion. The two chirped RC pulses are defined based on (3) with $N=2$. The parameters of the pulses are, $T_{0}=1.374 \mathrm{ps}$ which corresponds to the full width at half-maximum (FWHM) of 1 ps, $\alpha_{20}=-0.265 \mathrm{THz}^{2}$, and $P_{0}=10.6 \mathrm{~W} \quad\left(P_{0}=2\left|\beta_{20}\right| / \gamma / T_{0}^{2}\right)$. The peak power of initial pulse is optimized for the compression factor larger than 10 and energy ratio larger than $50 \%$. The corresponding initial dispersion length $L_{\mathrm{D}}$, nonlinear length $L_{\mathrm{N}}$, and chirp length $L_{\mathrm{C}}$ 
are $9.4 \mathrm{~m}, 4.7 \mathrm{~m}$ and $18.9 \mathrm{~m}$, respectively. Dispersion, nonlinear and chirp lengths with comparable lengths are suggested because the interaction between dispersion, nonlinearity and chirp can lead to efficient pulse compression. Figures. 1(a) and 1(b) illustrate the two chirped RC input pulses in both (a) linear and (b) logarithmic scales, respectively. Figs. 1(c) and 1(d) depict the output compressed pulse (solid line) and fitted hyperbolic secant pulse (dashed line) for a fiber length of $80 \mathrm{~m}$ in both (c) linear and (d) logarithmic scales, respectively. The fitted hyperbolic secant pulse is assumed to have the same FWHM and peak power as the compressed pulse. We define a single chirped RC pulse ( $N=1$ ) according to Eq. (2) with the same FWHM, peak power and chirp as that of two chirped RC pulses. The pulse widths of the input single chirped $\mathrm{RC}$ pulse and the

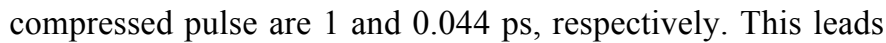
to a compression of 22.58. The peak power of the compressed pulse is 24.8 times greater than that of the initial pulse. It is essential to compute the energy ratio in order to understand the energy possessed by the compressed pulse. Therefore, it is defined as the ratio of the fitted hyperbolic secant pulse energy to the total input pulse energy and the fitted hyperbolic secant pulse is of the peak power and pulse width as the compressed pulse. The energy ratio of the compressed pulse is computed to be $60.5 \%$. From Figs. 1(c) and 1(d), we note that the compressed pulse attains nearly hyperbolic secant pulse profile. The curves in Figs. 1(e) and 1(f) are the spectra of the single chirped RC initial pulse (dashed line) and compressed pulse in $\left(t-t_{\mathrm{c}}\right) / T_{0} \in[-0.5,0.5]$ (solid line) in (e) linear and (f) logarithmic scales, respectively. Here $t_{\mathrm{c}}$ is the central position of compressed pulse. As shown in Figs. 1(e) and 1(f), obvious bandwidth broadening occurs owing to nonlinearity. In this case, the bandwidth broadening factor (BBF) is 13.55 . Another important parameter that quantities the quality of the compressed pulses is the time-bandwidth product (TBP). TBP of the compressed pulse in $\left(t-t_{\mathrm{c}}\right) / T_{0} \in[-0.5,0.5]$ is 0.3149 which is very close to the value of transform-limited hyperbolic secant pulse of 0.315 . Fig. 1(g) depicts the temporal evolution of compressed pulse at various stages of $(0$ $\mathrm{m}, 23 \mathrm{~m}, 40 \mathrm{~m}, 60 \mathrm{~m}, 80 \mathrm{~m})$ the compression process. Here we define the combination length as the fiber length when the peak power of the pedestal is less than $10 \%$ of the peak power of the compressed pulse. From Fig. 1(g), it is clear that the two chirped $\mathrm{RC}$ pulses coalesce into a single pulse at $23 \mathrm{~m}$ which is the combination length for the two chirped RC pulse compression. Then, the resulting combined pulse does undergo the self-similar pulse compression for a distance of 57 $\mathrm{m}$ till it reaches the end of the optical fiber of $80 \mathrm{~m}$. Because of the stimulated Raman scattering, there are temporal and spectral shifts in the output pulse as illustrated in Figs 1(c) and 1(e). Fig. 1(h) represents the compression factor of the two chirped RC pulse compression (solid line) for a length of $57 \mathrm{~m}$ when the combination process is just over and the dashed lines indicate the compression factor of the self-similar compression with the same fiber parameters $\exp (\sigma \times(z-23))$. The FWHM of the pulse at the fiber length of $23 \mathrm{~m}$ is $0.82 \mathrm{ps}$, and the
FWHM for the final $57 \mathrm{~m}$ is $0.044 \mathrm{ps}$ corresponding to the compression factor of 18.6 , which is close to the value 20.5 in the self-similar pulse compression. Thus, in the multi-pulse compression considered here, multiple input pulses first coalesce into a single pulse and then this single pulse undergoes nearly self-similar pulse compression.

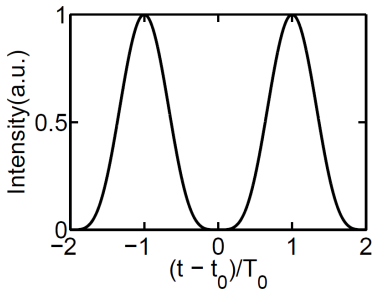

(a)

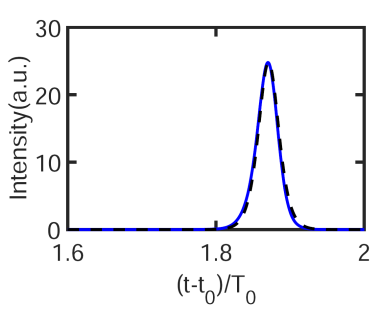

(c)

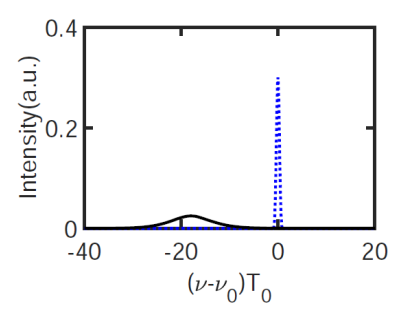

(e)

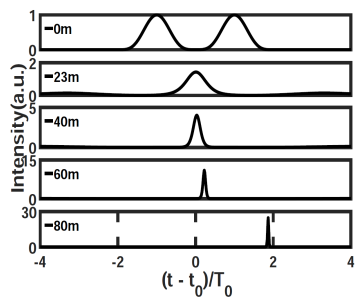

(g)

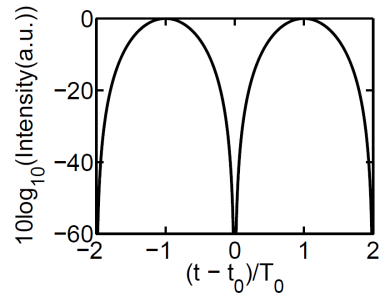

(b)

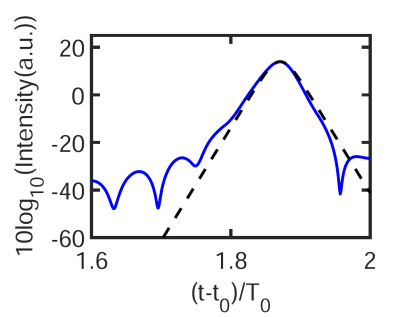

(d)

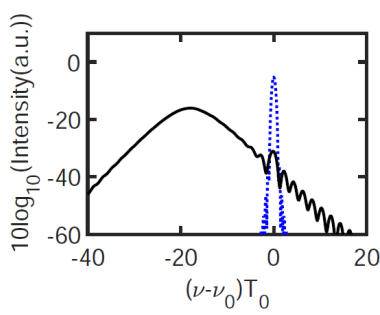

(f)

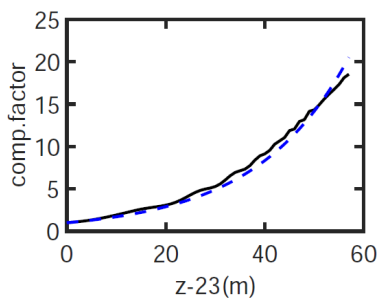

(h)
Fig. 1. Two chirped RC input pulses in (a) linear and (b) logarithmic scales. The compressed pulse (solid line) at fiber length of $80 \mathrm{~m}$ and fitted hyperbolic secant pulse (dashed line) in both (c) linear and (d) logarithmic scales. Spectra of the single chirped RC initial pulse (dashed line) and compressed pulse at 80 $\mathrm{m}$ in $\left(t-t_{\mathrm{c}}\right) / T_{0} \in[-0.5,0.5]$ (solid line) in both (e) linear and (f) logarithmic scales. (g) Temporal evolution at $0,23 \mathrm{~m}, 40 \mathrm{~m}, 60 \mathrm{~m}, 80 \mathrm{~m}$ (from top to bottom). (h) The compression factor of the two chirped RC compression (solid line) and corresponding compression factor (dashed line) of self-similar compression.

We also look into the property of the pulse at the combination length. Figures 2(a) and 2(b) show the combined pulse at $23 \mathrm{~m}$ in both linear and logarithmic scales, where the solid line and dashed line represent the combined pulse and fitted hyperbolic secant pulse, respectively. The resulting combined pulse nearly maintains the hyperbolic secant pulse shape, and contains $64.4 \%$ of input pulses' energy. Besides, we find that the 
FWHM of the combined pulse is $0.82 \mathrm{ps}$ and hence it undergoes a slight compression when compared to input pulse of width 1 ps. The TBF of the combined pulse is 0.599 which is close to that of the single pulse in the input pulse train $(0.525)$.

The main reason for the combination of the multiple pulses into a single pulse is owing to the common chirp profile shared by all the input multiple pulses. That is, the different spectral components in the multiple pulses do undergo almost the same phase shift at a particular distance. Consequently, the spectral components, which acquire like phase shift, are coalesced into a single pulse. The distance at which this process takes place is referred to as combination length. Then, the resulting combined pulse does undergo compression under the influence of nonlinear effects. Finally, it evolved into a hyperbolic secant pulse shape from the initial RC pulse shape.

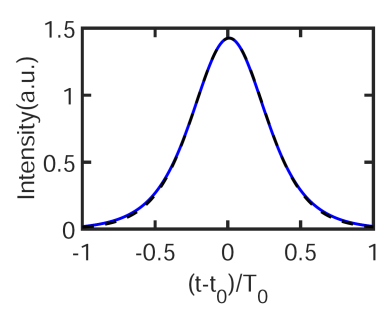

(a)

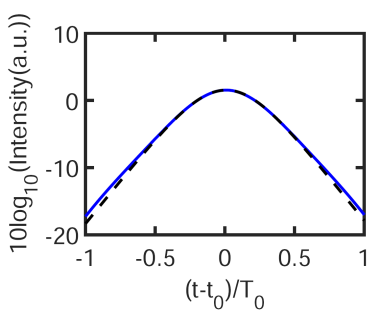

(b)
Fig. 2. The combined pulse (solid line) and fitted hyperbolic secant pulse (dashed line) at $23 \mathrm{~m}$ in (a) linear and (b) logarithmic scales.

\section{Three, Four and Five Chirped RC Pulses}

Here we consider the compression of three, four and five chirped RC input pulses. The three and five pulses are chosen based on Eq. (2) and four pulses are according to Eq. (3). The three, four and five chirped RC input pulses are chosen to have the same $T_{0}$ and $\alpha_{20}$ as the two chirped RC pulses in Sec. III.A. However, the initial peak power $P_{0}$ is different for different pulses of different numbers, that is, $P_{0}=5.3 \mathrm{~W}, 2.91$ $\mathrm{W}$, and $1.85 \mathrm{~W}$ (which corresponds to $1,0.55$, and 0.35 , respectively, according to the relation, $P_{0} /\left(\left|\beta_{20}\right| / \gamma / T_{0}{ }^{2}\right)$ for $N=$ 3,4 and 5, respectively. Similarly, the peak power of initial pulse is optimized for the compression factor larger than 10 and energy ratio larger than $50 \%$. Figure 3 (a) shows the input of three, four and five (top to bottom) chirped RC pulses. Figure 3(b) gives the compression factor for three (dots), four (circles) and five (crosses) chirped RC pulses along the fiber propagation direction. For a given fiber length of $80 \mathrm{~m}$, we observe that the fiber length required to combine multiple pulses, i.e., the combination length gets increased as the number of pulses increases. Consequently, the compression factor becomes smaller for higher pulse numbers. Figures 3(c) and 3(d) represent the compressed self-similar USPs at $80 \mathrm{~m}$ for three (dotted-dashed line), four (dashed line) and five (solid line) chirped RC pulses in (c) linear and (d) logarithmic scales, respectively. Figures 3(e) and 3(f) portray the spectra of the compressed pulse in $\left(t-t_{\mathrm{c}}\right) / T_{0} \in[-0.5,0.5]$ for three (dotted-dashed line), four (dashed line) and five (solid line) chirped RC pulses in (e) linear and (f) logarithmic scales. From Figs. 3(e) and 3(f), it is obvious that the bandwidth broadening is relatively less for the USPs that are resulted from the higher numbers of input pulses since they undergo less compression. Distinct temporal and spectral shifts occur for the input pulses with lower numbers due to the stimulated Raman scattering. To gain the much insight in these compression processes, in Table I, we compare all the important out characteristics, namely, compression factor, BBF, TBP, peak power, energy ratio, and combination length of the compressed self-similar USPs that are resulted from different numbers of chirped RC pulses $(N=2,3,4$, and 5). According to Table I, we find that both the compression factor and bandwidth broadening factor (BBF) decrease with the pulse number. This is because the combination length increases with the pulse number. Therefore, the combined pulses do undergo compression only for a short length of a fiber. In addition to this, we compute the TBP of the compressed USPs and it is close to transform-limited value of 0.315 . Undoubtedly, these two output characteristics imply that the generated high energy self-similar USPs are almost free from chirp. These are desirable characteristics of any USPs which are generated from pulse compression technique. Further, bandwidth broadening does occur which signify the nonlinear compression process. Finally, we calculate the energy ratio that stays around $60 \%$ for different input pulse numbers. This indirectly implies that the generated USPs possess high energy.

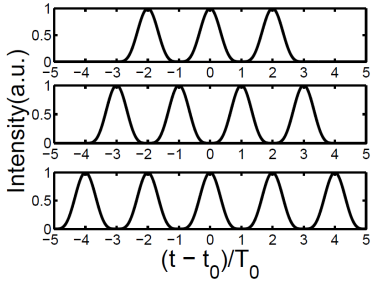

(a)

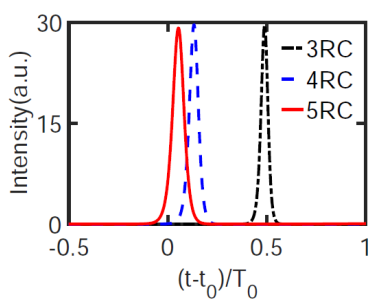

(c)

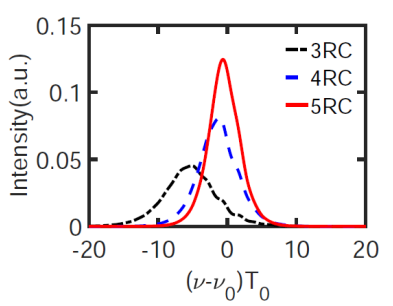

(e)

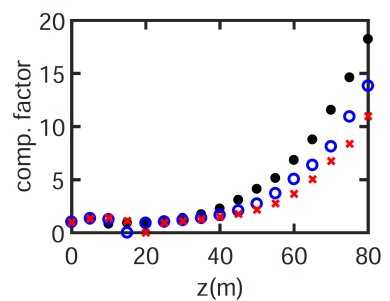

(b)

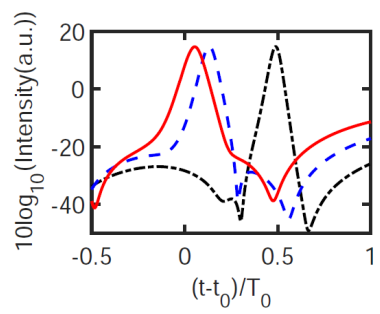

(d)

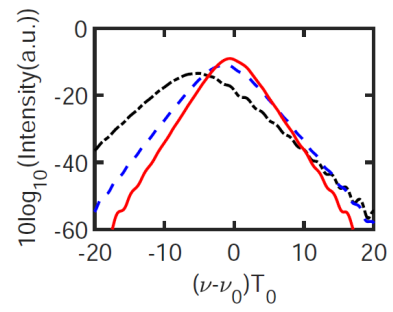

(f)
Fig. 3. (a) Input three, four, and five chirped RC pulses (from top to bottom). (b) The compression factor of three (dots), four (circles) and five (crosses) chirped RC pulses along the fiber propagation direction. Output pulses at fiber 
length of $80 \mathrm{~m}$ for three (dot dashed line), four (dashed line) and five (solid line) chirped RC pulses in (c) linear and (d) logarithmic scales. Spectra of the output pulses at $80 \mathrm{~m}$ in $\left(t-t_{\mathrm{c}}\right) / T_{0} \in[-0.5,0.5]$ for three (dot dashed line), four (dashed line), five (solid line) pulses in both (e) linear and (f) logarithmic scales.

TABLE I

COMPARISON OF VARIOUS OUTPUT CHARACTERISTICS, NAMELY, COMPRESSION FACTOR, BBF, TBP, PEAK POWER, ENERGY RATIO AND COMBINATION LENGTH FOR N CHIRPED RC PULSES

\begin{tabular}{c|c|c|c|c}
\hline & $N=2$ & $N=3$ & $N=4$ & $N=5$ \\
\hline Comp. factor & 22.58 & 18.22 & 13.82 & 10.97 \\
\hline BBF & 13.55 & 10.50 & 8.24 & 6.64 \\
\hline TBP & 0.315 & 0.303 & 0.313 & 0.318 \\
\hline $\begin{array}{c}\text { Peak Power } \\
\text { (W) }\end{array}$ & 262.74 & 156.75 & 86.33 & 53.98 \\
\hline Energy Ratio & $60.5 \%$ & $59.6 \%$ & $59.1 \%$ & $58.6 \%$ \\
\hline $\begin{array}{c}\text { Combination } \\
\text { length (m) }\end{array}$ & 23 & 33 & 44 & 52 \\
\hline
\end{tabular}

\section{Chirped Hyperbolic SECANT Pulses}

\section{A. Input Pulse Shape}

Having discussed the generation of high energy USPs from $\mathrm{RC}$ pulses, in this section, we intend to investigate the generation of similar USPs by injecting the chirped hyperbolic secant pulse profiles. Here, the chirped hyperbolic secant pulses are described by the following form of:

$$
\sum_{m=-N}^{N} \sqrt{P_{0}} \operatorname{sech}\left(t / T_{0}+m \xi\right) \exp \left(i \alpha_{20} t^{2} / 2\right),
$$

(odd pulse number, pulse number: $2 N+1$ )

$\sum_{m=-N}^{N-1} \sqrt{P_{0}} \operatorname{sech}\left(t / T_{0}+\xi / 2+m \xi\right) \exp \left(i \alpha_{20} t^{2} / 2\right)$,

(even pulse number, pulse number: $2 N$ )

where $\alpha_{20}, P_{0}$, and $T_{0}$ are the initial chirp, initial peak power and initial pulse width parameters, respectively. Here, $\xi$ is the separation between two neighboring hyperbolic secant pulses. In this case, the fiber parameters are assumed to be the same as in Sec. III.

\section{B. Two Chirped Hyperbolic Secant Pulses}

Now, we discuss the compression of chirped multiple hyperbolic secant pulses. To start with, we consider the two chirped hyperbolic secant pulses according to (5). The pulses parameters are $N=1, \xi=5 T_{0} \mathrm{ps}, T_{0}=0.908 \mathrm{ps}$ which corresponds to FWHM of $1.6 \mathrm{ps}, \alpha_{20}=-0.265 \mathrm{THz}^{2}$, and $P_{0}=3.0 \mathrm{~W}\left(P_{0}=0.25\left|\beta_{20}\right| / \gamma / T_{0}^{2}\right)$. Similarly, the peak power of initial pulse is optimized for the compression factor larger than 10 and energy ratio larger than $50 \%$. Using these pulse parameters as well as fiber parameters, the length scales, namely, $L_{\mathrm{D}}, L_{\mathrm{N}}, L_{\mathrm{C}}$ are $4.1 \mathrm{~m}, 16.7 \mathrm{~m}$ and $18.9 \mathrm{~m}$, respectively. Figures 4 (a) and 4(b) portray two chirped hyperbolic secant input pulses in (a) linear and (b) logarithmic scales,

respectively. Figsures 4(c) and 4(d) depict the compressed high energy USP at the fiber length of $80 \mathrm{~m}$ (solid line) and the fitted hyperbolic secant pulse (dashed line) in both (c) linear and (d) logarithmic scales, respectively. We define the single chirped hyperbolic secant pulse $(N=0)$ using Eq. (4) with the same $T_{0}, P_{0}$ and $\alpha_{20}$ as that of two chirped hyperbolic secant pulses. When a chirped hyperbolic secant pulse with a width of $1.6 \mathrm{ps}$ is launched, it is temporally compressed down to $58 \mathrm{fs}$, resulting in a compression factor of 27.78. Hence, the peak power of the compressed pulse gets increased and is greater 47 times than the initial pulses. As we deal with the high energy USPs, we also calculate the energy ratio of the compressed pulse and is found to be $79.6 \%$. From Figs. 4(c) and 4(d), we note that the compressed pulse is nearly hyperbolic secant pulse shape. Figures 4(e) and 4(f) show the spectra of the single chirped hyperbolic secant initial pulse (dashed line) and compressed pulse in $\left(t-t_{\mathrm{c}}\right) / T_{0} \in[-0.5$, 0.5 ] (solid line) in (e) linear and (f) logarithmic scales, respectively. As depicted in Figs. 4(e) and 4(f), the bandwidth broadening occurs owing to the nonlinear effect and the BBF is 22.68. The TBP of the compressed pulse is 0.320 in $\left(t-t_{\mathrm{c}}\right) / T_{0}$ $\in[-0.5,0.5]$. Figure $4(\mathrm{~g})$ represents the dynamics of the compressed pulse at various stages of the fiber during the compression process. The well separated initial pulses are combined at a fiber length of $21 \mathrm{~m}$. The FWHM of the pulse at the combination length is $1.00 \mathrm{ps}$. This combined pulse does undergo self-similar pulse compression for the remaining length of $59 \mathrm{~m}$. Fig. 4(h) depicts the compression factor of the two chirped hyperbolic secant pulses after combination process (solid line) and the corresponding compression factor of the self-similar compression (dashed line). As shown in Fig. 4(h), the compression factor after the combination process (for $59 \mathrm{~m}$ ) is 17.52 and the compression factor in the self-similar approximation is $\exp (\sigma \times(L-21))=22.81$. We note that the compression factor due to the former one is close to the direct self-similar pulse compression.

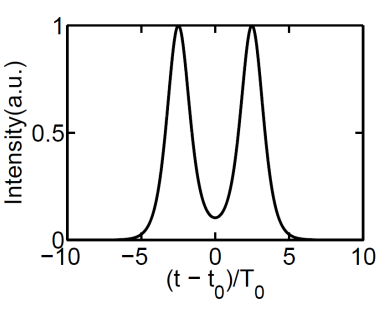

(a)

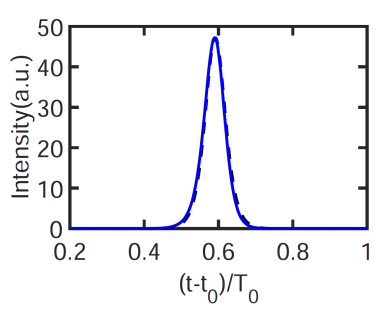

(c)

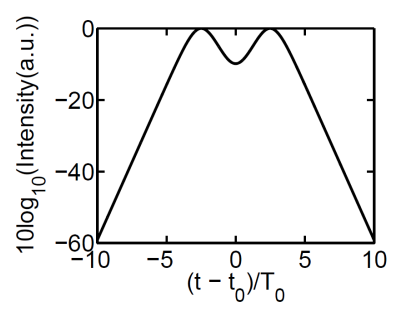

(b)

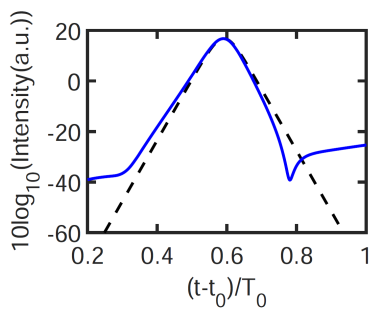

(d) 


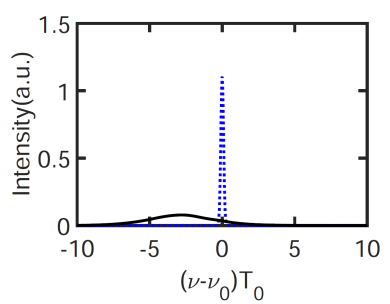

(e)

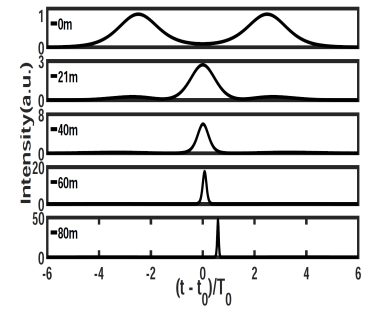

(g)

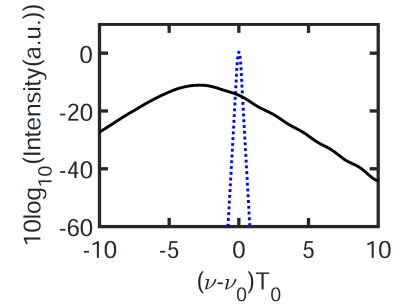

(f)

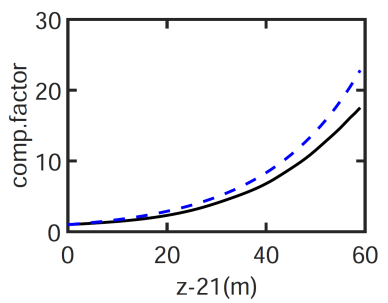

(h)
Fig. 4. Two chirped hyperbolic secant input pulses in (a) linear and (b) logarithmic scales. The compressed pulse (solid line) at fiber length of $80 \mathrm{~m}$ and fitted hyperbolic secant pulse (dashed line) in both (c) linear and (d) logarithmic scales, respectively. Spectra of the single chirped hyperbolic secant initial pulse (dashed line) and compressed pulse at $80 \mathrm{~m}$ in $\left(t-t_{\mathrm{c}}\right) / T_{0} \in$ $[-0.5,0.5]$ (solid line) in both (e) linear and (f) logarithmic scales. The (g) temporal evolution and (h) compression factor of the two chirped hyperbolic secant pulses compression (solid line) and the corresponding compression factor of the self-similar compression (dashed line) after the combination process.

\section{Three, Four and Five Chirped Hyperbolic Secant Pulses}

In this sub-section, we extend the above analysis for three, four and five chirped hyperbolic secant pulses. The pulse parameters are same as the two chirped hyperbolic secant pulses in Sec. IVA except the peak power. The peak power of initial pulse is optimized for the compression factor larger than 10 and energy ratio larger than $50 \%$. As a result, different initial peak power $P_{0}$ are selected for different pulses of different numbers, i.e., $P_{0}=1.214,0.728$, and $0.486 \mathrm{~W}$ for the pulses of 3,4 , and 5 , respectively. The input pulses of three and five pulses are based on Eq. (4) with $N=1$ and 2, respectively. The four pulses are in the form of Eq. (5) with $N$ $=2$. Fig. 5(a) illustrates the input of three, four and five chirped hyperbolic secant pulses. The evolution of compression factor of the three (dots), four (circles) and five (crosses) chirped hyperbolic secant pulses compression at various stages of the propagation in the exponentially decreasing fiber is depicted in Fig. 5(b). As in the previous case, here also we find that the compression factor decreases with the pulse number. Figures 5(c) and 5(d) represent the intensity of the generated USPs that are resulted from three (dot-dashed line), four (dashed line) and five (solid line) chirped hyperbolic secant pulses in (c) linear and (d) logarithmic scales, respectively. Further, we also provide the spectra of the compressed pulses in $\left(t-t_{\mathrm{c}}\right) / T_{0} \in[-0.5,0.5]$ for three (dot-dashed line), four (dashed line) and five (solid line) chirped hyperbolic secant pulses in terms of linear scale in Fig. 5(e) linear and logarithmic scales in Fig. 5(f). From Figs. 5(c) to 5(f), we observe the temporal and spectral shifts in the output pulse owing to the stimulated Raman scattering. Table
II gives the comparison of the compression factor, BBF, TBP, peak power, energy ratio and combination length for $N=2,3$, 4 , and 5 chirped hyperbolic secant pulses with the separation parameter of $5 T_{0}$. As in Sec. III, the compression factor and BBF decrease with input pulse number, while the combination length increases with input pulse number. For different input pulse numbers, the energy ratios stay around $78 \%$ and hence the generated USPs possesses high energy.

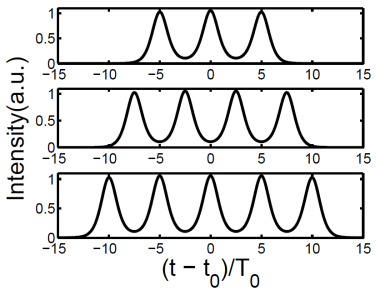

(a)

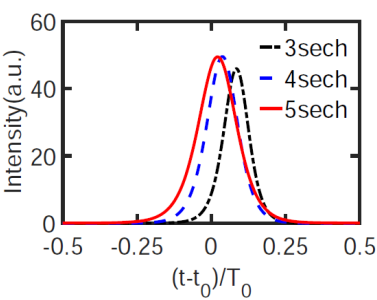

(c)

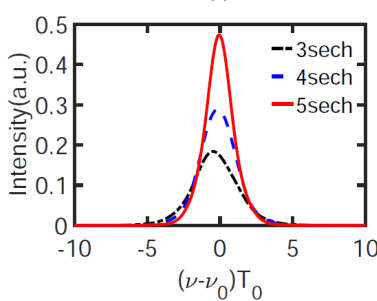

(e)

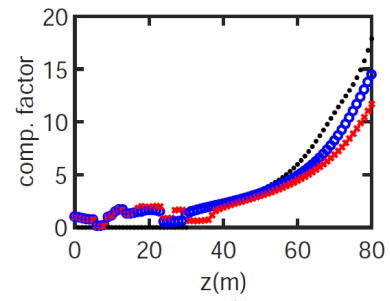

(b)

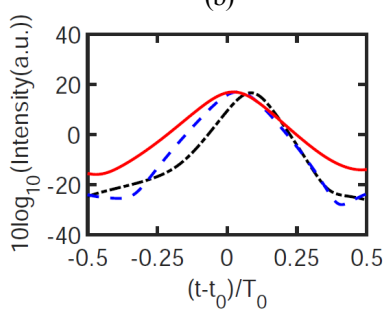

(d)

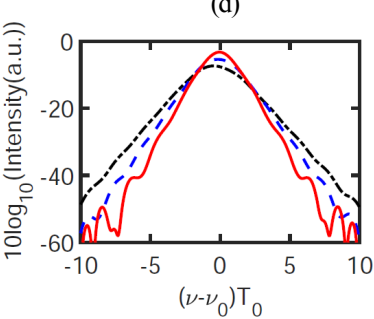

(f)
Fig. 5. (a) Input of three, four and five (from top to bottom) chirped hyperbolic secant pulses. (b) The compression factor of three (dots), four (circles) and five (crosses) chirped hyperbolic secant pulses compression along the fiber propagation direction. Output pulses at fiber length of $80 \mathrm{~m}$ for three (dot-dashed line), four (dashed line) and five (solid line) hyperbolic secant pulses in (c) linear and (d) logarithmic scales. Spectra of output pulses at $80 \mathrm{~m}$ in $\left(t-t_{\mathrm{c}}\right) / T_{0} \in[-0.5,0.5]$ for three (dot-dashed line), four (dashed line), five (solid line) pulses in both (e) linear and (f) logarithmic scales.

TABLE II

COMPRESSION Factor, BBF, TBP, PEAK POWER, ENERgy RATIO AND COMBINATION LENGTH FOR N CHIRPED HYPERBOLIC SECANT PULSES COMPRESSION WITH SEPARATION OF 5

\begin{tabular}{c|c|c|c|c}
\hline & $N=2$ & $N=3$ & $N=4$ & $N=5$ \\
\hline Comp. factor & 27.78 & 17.87 & 14.46 & 11.68 \\
\hline BBF & 22.68 & 14.74 & 12.69 & 9.21 \\
\hline TBP & 0.320 & 0.324 & 0.344 & 0.309 \\
\hline Peak Power (W) & 141.57 & 55.73 & 36.05 & 24.03 \\
\hline Energy Ratio & $79.6 \%$ & $78.5 \%$ & $77.7 \%$ & $76.3 \%$ \\
\hline $\begin{array}{c}\text { Combination } \\
\text { length (m) }\end{array}$ & 21 & 31 & 39 & 47 \\
\hline
\end{tabular}

Figure 6 depicts the variation of (a) compression factor, (b) energy ratio and (c) combination length against the pulse-to-pulse separation $\xi$ for three input pulses. Here, the fiber and pulse parameters are the same in Fig. 5. We find that both the compression factor and energy ratio decrease with the 
pulse-to-pulse separation. However, the combination length increases. Thus, the numerical results in Fig. 6 support that the proposed compression scheme works well even if the pulse-to-pulse separation of the input pulses is varied.

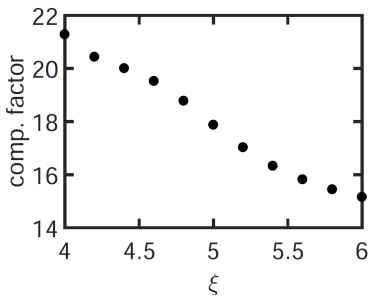

(a)

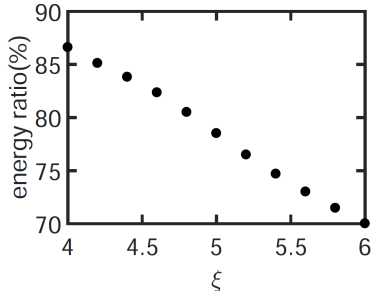

(b)

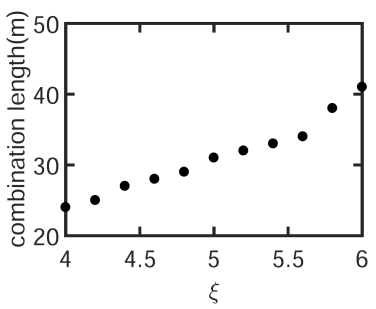

(c)

Fig. 6. The (a) compression factor, (b) energy ratio and (c) combination length versus different pulse-to-pulse separation.

Figure 7 provides a detailed study about the combination length and compression factor, considering different input pulse shape, number of input pulses, input pulse width. The peak power is the optimized peak power for input $\mathrm{RC}$ and hyperbolic secant pulses. Figures 7(a) and 7(b) show the combination length and corresponding compression factor for input RC pulses, and Figs. 7(c) and 7(d) are the results for input hyperbolic secant pulses. The cross, circle, dot, diamond and plus symbols in Figs. 7(a) and 7(b) represent initial FWHM of $0.8 \mathrm{ps}, 0.9 \mathrm{ps}, 1.0 \mathrm{ps}, 1.1 \mathrm{ps}, 1.2 \mathrm{ps}$ for combining 2, 3, 4, 5 chirped RC pulses. Similarly, the cross, circle, dot, diamond, plus and square symbols in Figs. 7(c) and 7(d) indicate initial FWHM of $1.0 \mathrm{ps}, 1.2 \mathrm{ps}, 1.4 \mathrm{ps}, 1.6 \mathrm{ps}, 1.8 \mathrm{ps}$, 2.0 ps for combining 2, 3, 4, 5 chirped hyperbolic secant pulses. For both input RC and hyperbolic secant pulses, the combination length increases with input pulse width for given pulse shape and number of pulses as shown in Figs. 7(a) and 7(c). Similarly, for given pulse width, the combination length increases but the compression factor decreases with the input number of pulses as shown in Figs. 7(a-d).

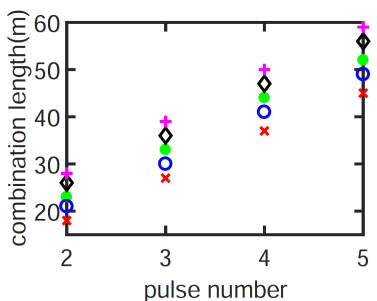

(a)

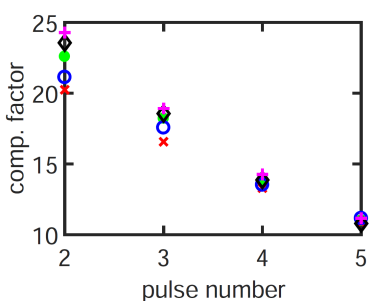

(b)

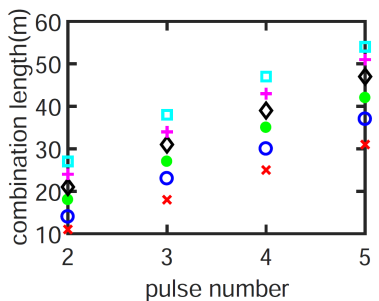

(c)

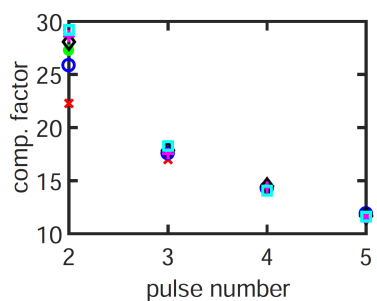

(d)
Fig. 7. (a) The combination length and (b) compression factor for the initial FWHM of 0.8 (crosses), 0.9 (circles), 1.0 (dots), 1.1 (diamonds), 1.2 (pluses) with 2, 3, 4, 5 chirped RC pulses; (c) The combination length and (d) compression factor for the initial FWHM of 1.0 (crosses), 1.2 (circles), 1.4 (dots), 1.6 (diamonds), 1.8 (pluses), 2.0 (squares) with 2, $3,4,5$ chirped hyperbolic secant pulses.

\section{Discussions}

\section{A. Significance of the initial pulse chirp}

The self-similar compression in [13-16] is based on the chirped soliton solution to the NLS equation with exponentially decreasing dispersion. In self-similar compression, the evolution of self-similar soliton is mainly governed by the interplay of the pulse chirp with the fiber dispersion and nonlinearity all along the propagation [16]. The initial chirp plays a critical role in the self-similar compression. In this Section, we study the significance of the initial chirp in the multi-pulse compression. We consider the compression of three chirp-free RC and hyperbolic secant pulses and compare the results with that of the chirped pulses. The chirp-free RC pulses are in the form of $\sqrt{P_{0}} / 2\left(1+\cos \left(\pi t / T_{0}\right)\right), t / T_{0} \in[-N, N]$ where $N, T_{0}$, $P_{0}$ are the same as three chirped RC pulses in Sec. III B. The chirp-free hyperbolic secant pulses are in the form of $\sum_{m=-N}^{N} \sqrt{P_{0}} \operatorname{sech}\left(t / T_{0}+m \xi\right)$ where $\xi$ is 5 and $N, T_{0}, P_{0}$ are the same as three chirped hyperbolic secant pulses in Sec. IV B. Figures 8(a) and 8(b) depict the compressed pulse of three chirp-free RC pulses (solid line) and three chirped RC pulses (dashed line) for a fiber length of $80 \mathrm{~m}$ in (a) linear and (b) logarithmic scales, respectively. Figures 8 (a) and 8 (b) show the significant role played by the initial pulse chirp for better compression as the chirped RC pulses (dashed line) go through more compression than the chirp-free RC pulses (solid line). Figures 8(c) and 8(d) are the intensity contour plots of the evolution of three chirp-free RC and chirped RC input pulses along the fiber. For the chirped input RC pulses, the energy ratio is $59.6 \%$, and the compression factor is 18.22 . For the chirp-free $\mathrm{RC}$ input pulses, the energy ratio is only $30.1 \%$, and the compression factor is 9.47. The compressed pulses of the three chirp-free hyperbolic secant pulses (solid line) and three chirped hyperbolic secant pulses (dashed line) are given in linear (Fig. 8(e)) and logarithmic (Fig. 8(f)) scales, respectively. Figures $8(\mathrm{~g})$ and $8(\mathrm{~h})$ are the intensity contour plots of the evolution of three chirp-free hyperbolic secant and three chirped hyperbolic secant input pulses along the fiber. As shown in Fig. 8(g), three chirp-free hyperbolic secant pulses could not combine into one single pulse. Thus, it is clear that the initial pulse chirp plays an important role in the pulse compression scheme proposed here. 


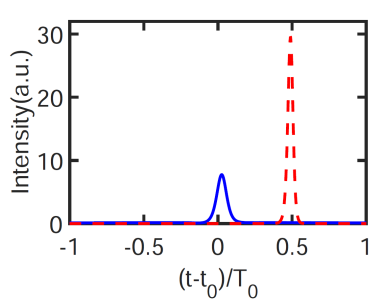

(a)

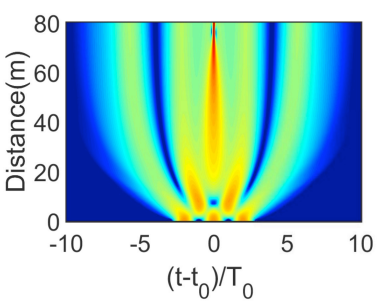

(c)

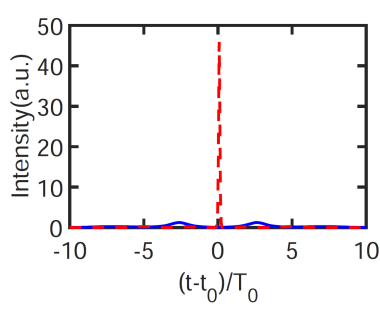

(e)

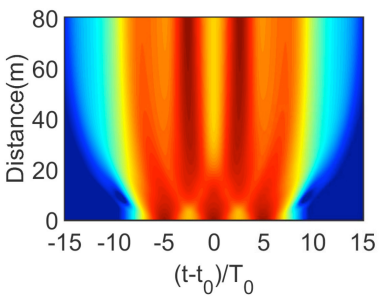

(g)

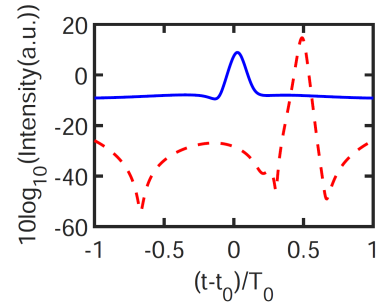

(b)

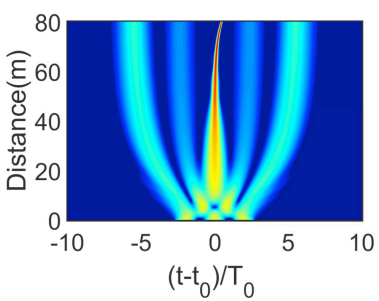

(d)

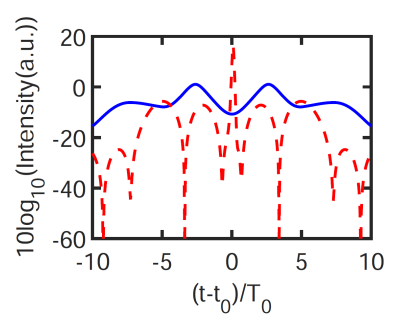

(f)

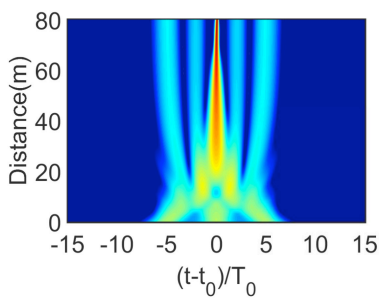

(h)
Fig. 8. The compressed pulse of three chirp-free RC pulses (solid line) and three chirped RC pulses (dashed line) at the fiber length of $80 \mathrm{~m}$ is depicted in (a) linear and (b) logarithmic scales, respectively. (c) and (d) are the corresponding evolution of the three (c) chirp-free and (d) chirped RC input pulses along the fiber. The compressed pulse of three chirp-free hyperbolic secant pulses (solid line) and three chirped hyperbolic secant pulses (dashed line) at the fiber length of $80 \mathrm{~m}$ is depicted in (e) linear and (f) logarithmic scales. (g) and (h) are the corresponding evolution of the three $(\mathrm{g})$ chirp-free and (h) chirped hyperbolic secant input pulses along the fiber.

\section{B. Variation of the initial pulse chirp or dispersion decay rate}

According to the self-similar analysis, decay rate of the dispersion is given by $\sigma=\alpha_{20} \beta_{20}$. Here, $\alpha_{20}, \beta_{20}$ and $\sigma$ are the initial pulse chirp, initial fiber dispersion and dispersion decay rate, respectively. In this sub-section, we will study the performance of the proposed pulse compression scheme by varying the initial pulse chirp or dispersion decay rate.

The initial pulse chirp is calculated by the relation $\sigma=\alpha_{20} \beta_{20}$ based on a condition in the self-similar pulse compression. Here, the initial pulse chirp is varied by $\pm 20 \%$ and other pulse and fiber parameters remain the same as in Fig. 1. When the initial pulse chirp is carefully chosen as $\alpha_{20}=\sigma / \beta_{20}$, the corresponding pulse compression factor is 22.58. However, if the initial chirp is less $20 \%\left(\alpha_{20}=0.8 \sigma / \beta_{20}\right)$ or greater than $20 \%\left(\alpha_{20}=1.2 \sigma / \beta_{20}\right)$ of the original value, then the resulting compression factor turns out to be 22.01 or 22.34 , which is quite close to that of the ideal condition $\alpha_{20}=\sigma / \beta_{20}$. The energy ratio also maintains around $60 \%$ when the initial pulse chirp is varied. The final compressed pulse (solid line) and fitted hyperbolic secant pulse (dashed line) for two chirped RC input pulses are shown in Fig. 9 when $\alpha_{20}=0.8 \sigma / \beta_{20}$ in Figs. 9(a, c) and when $\alpha_{20}=1.2 \sigma / \beta_{20}$ in Figs. 9(b, d). Here, the compressed pulse maintains the hyperbolic secant pulse shape when the initial pulse chirp is varied. By carrying out extensive simulations, we have found that the change of compression factor is generally less than $5 \%$ when the initial pulse chirp is varied by $\pm 20 \%$.

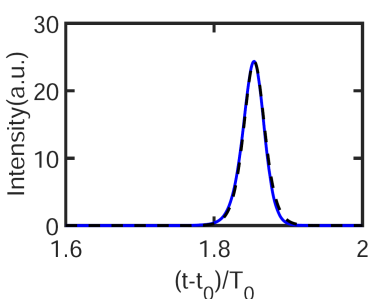

(a)

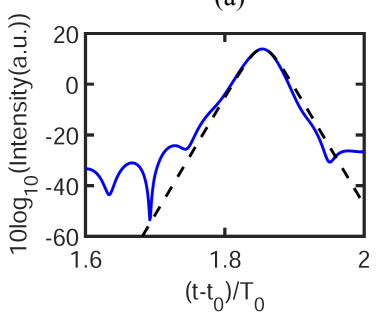

(c)

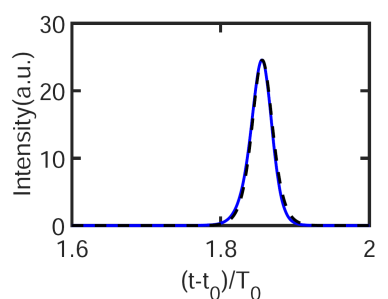

(b)

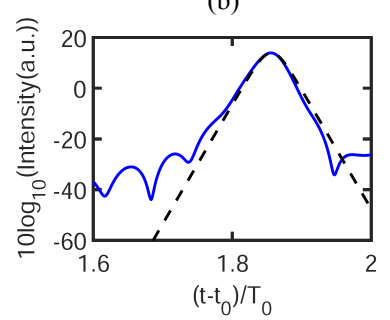

(d)
Fig. 9. The compressed pulse (solid line) at fiber length of $80 \mathrm{~m}$ and fitted hyperbolic secant pulse (dashed line) for two chirped RC input pulses in linear (a, b) and logarithmic scales (c, d) with $\alpha_{20}$ equals to (a, c) $0.8 \sigma / \beta_{20}$ and (b, d) $1.2 \sigma / \beta_{20}$.

Secondly, the dispersion decay rate is varied by $\pm 20 \%$ and the other pulse and fiber parameters remain the same as in Fig. 1. When the dispersion decay rate is carefully chosen as $\sigma=\alpha_{20} \beta_{20}$, the corresponding pulse compression factor is 22.58. When $\sigma=0.8 \alpha_{20} \beta_{20}$, the resulting compression factor is 11.75 and the energy ratio is $64 \%$. When $\sigma=1.2 \alpha_{20} \beta_{20}$, the resulting compression factor is 42.91 and the energy ratio is only $44 \%$. The final compressed pulse (solid line) and fitted hyperbolic secant pulse (dashed line) for two chirped RC input pulses are shown in Fig. 10 when $\sigma=0.8 \alpha_{20} \beta_{20}$ in Figs. 10(a, c) and when $\sigma=1.2 \alpha_{20} \beta_{20}$ in Figs. 10(b, d). If the dispersion decay rate is reduced, the compressed pulse nearly maintains the hyperbolic secant pulse, but the compression factor is much less. If the dispersion decay rate is increased, the compression factor becomes much higher, but the compressed pulse deviates from the hyperbolic secant pulse shape. Thus, based on these results, the proposed scheme is less sensitive to the variation of initial pulse chirp. 

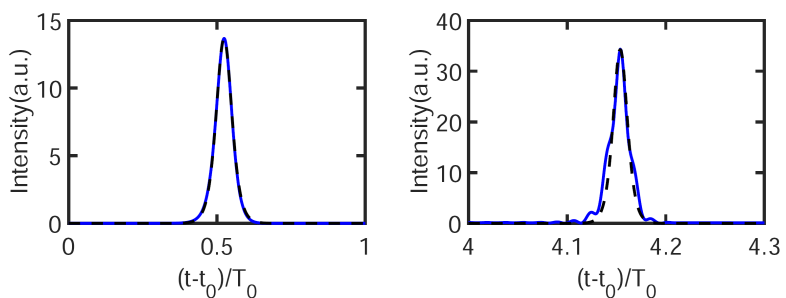

(a)

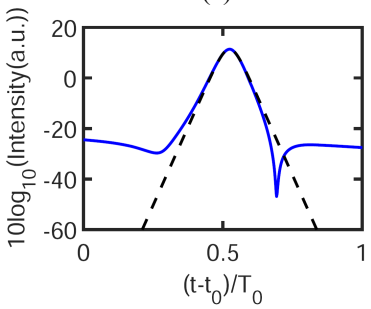

(c)

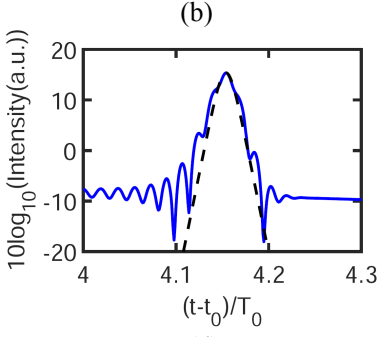

(d)

Fig. 10. The compressed pulse (solid line) at fiber length of $80 \mathrm{~m}$ and fitted hyperbolic secant pulse (dashed line) for two chirped RC input pulses in linear $(\mathrm{a}, \mathrm{b})$ and logarithmic scales (c, d) with $\sigma$ equals to (a, c) $0.8 \alpha_{20} \beta_{20}$ and (b, d)

$1.2 \alpha_{20} \beta_{20}$.

\section{CONCLUSION}

In conclusion, we have studied the multi-pulse combination into a single pulse and then compression using two different pulse profiles, namely, RC and hyperbolic secant pulses in nonlinear optical fibers with the exponentially decreasing dispersion. The input pulse train is firstly modulated using a phase modulator in which each modulation cycle could cover two, three, four, or five pulses. The resulting pulses possess the same linear chirp, could combine into a single pulse, which, in turn, did experience effective pulse compression in the nonlinear fiber with the exponentially decreasing dispersion. It turns out the final compressed pulse contains majority of the input pulses energy to become an ultrashort high-energy single pulse. In the case of initial hyperbolic secant shaped pulses combination and compression, the final high-energy pulse is almost chirp free. We have also studied the compression of $2,3,4$, and 5 pulses with both the pulse profiles. The energy ratio of the compressed pulses for both RC and hyperbolic secant stays almost the same and the compression factor decreases with the increase of the number of pulses. For the hyperbolic secant input pulses, both the compression factor and energy ratio decrease while the combination length increases with the pulse-to-pulse separation. For a given pulse shape (RC or hyperbolic secant) and number of pulses, the combination length increases with input pulse width. The combination length increases and the compression factor decreases with the increase in the input number of pulses for the same input pulse width. Besides, we have also compared the compression of chirped and chirp-free input pulses in order to understand the influence of initial chirp. By dint numerical simulation, we have inferred that the multiple hyperbolic secant pulses do not combine into a single pulse and hence no compression at all, which implies the importance of the initial pulse chirp. We have also varied the initial pulse chirp or dispersion decay rate, and find the suggested scheme is less sensitive to the change of initial

pulse chirp. The proposed compression scheme is a good candidate for high energy ultrashort pulse generation.

\section{ACKNOWLEDGMENT}

This work was supported by the National Natural Science Foundation of China (No. Project 61675008).

\section{REFERENCES}

[1] M. E. Fermann, A. Galvanauskas, and G. Sucha, Ultrafast Laser: Technology and applications, CRC Press, 2002.

[2] J. Meijer, J. Materials Processing Technol., vol. 149, pp. 2-17, 2004.

[3] G. P. Agrawal, Applications of Nonlinear Fiber Optics, Academic Press, 2001.

[4] J. Limpert, F. Röser, T. Schreiber, and A. Tünnermann, IEEE J. Sel. Top. Quantum Electron., vol. 12, pp. 233-244, 2006.

[5] F. W. Wise, A. Chong, and W. H. Renninger, Laser \& Photonics Reviews, vol. 2, pp. 58-73 (2008)

[6] D. J. Richardson, J. Nilsson, and W. A. Clarkson, J. Opt. Soc. Am. B, vol. 27, pp. 63-92, 2010.

[7] L. Lombard, A. Azarian, K. Cadoret, P. Bourdon, D. Goular, G. Canat, V. Jolivet, Y. Jaouën, and O. Vasseur, Opt. Lett., vol. 36, pp. 523-525, 2011.

[8] S. V. Chernikov and P. V. Mamyshev, J. Opt. Soc. Am. B, vol. 8, pp. 1633-1641, 1991.

[9] K. C. Chan, and H. F. Liu, IEEE J. Quantum Electron., vol. 31, pp. 2226-2235, Dec. 1995.

[10] B. J. Eggleton, G. Lenz, and N. M. Litchinitser, Fiber \& Integrated Optics, vol. 19, pp. 383-421, 2000.

[11] M. D. Pelusi and H. F. Liu, IEEE J. Quantum Electron., vol. 33, pp. 1430-1439, 1997.

[12] J. D. Moores, Opt. Lett., vol. 21, pp. 555-557, 1996.

[13] V. I. Kruglov, A. C. Peacock, J. D. Harvey, and J. M. Dudley, J. Opt. Soc. Am. B, vol. 19, pp.461-469, 2002.

[14] V. I. Kruglov, A. C. Peacock, and J. D. Harvey, Phys. Rev. Lett., vol. 90, no. 11, pp. 113902-1-113902-4, Mar. 2003.

[15] J. M. Dudley, C. Finot, D. J. Richardson, and G. Millot, Nature Physics, vol. 3, pp. 597-603, 2007.

[16] Q. Li, K. Senthilnathan, K. Nakkeeran, and P. K. A. Wai, J. Opt. Soc. Am. B, vol. 26, pp. 432-443, 2009.

[17] Q. Li, P. K. A. Wai, K. Senthilnathan, K. Nakkeeran, J. Lightwave Technol., vol. 29, pp. 1293-1305, 2011.

[18] R. V. J. Raja, K. Senthilnathan, K. Porsezian and K. Nakkeeran, IEEE Journal of Quantum Electronics, vol. 46, pp. 1795-1803, 2010.

[19] I. E. Mansouri, J Fatome, C. Finot, M. Lintz, and S. Pitois. IEEE Photonics Technol. Lett., vol. 23, pp. 1487-1489, 2011.

[20] S. Olupitan, K. Senthilnathan, P. R. Babu, S. S. Aphale, and K. Nakkeeran, IEEE Photonics J., vol. 4, no. 5, pp. 1420-1437, Oct. 2012.

[21] S. Olupitan, K. Senthilnathan, P. R. Babu, R. V. J. Raja, S. S. Aphale, and K. Nakkeeran, J. Mod. Opt., vol. 60, no. 5, pp. 368-377, 2013.

[22] D. A. Korobko, O.G. Okhotnikov, and I. O. Zolotovskii, J. Opt. Soc. Am. B, vol. 30, pp. 2377-2386, 2013.

[23] Q. Li, K. Nakkeeran, and P. K. A. Wai, J. Opt. Soc. Am. B, vol. 31, pp. 1786-1792, 2014.

[24] W. Lu, Q. Li, and P. K. A. Wai, in Asia Communications and Photonics Conference 2014, Shanghai, China, 2014.

[25] W. Lu, Q. Li, and P. K. A. Wai, in Asia Communications and Photonics Conference 2015, Hong Kong, China, 2015.

[26] G. P. Agrawal, Nonlinear Fiber Optics, 4th ed., Academic Press, 2007.

[27] R. H. Stolen, J. P. Gordon, W. J. Tomlinson, and H. A. Haus, J. Opt. Soc. Am. B, vol. 6, pp. 1159-1166, 1989.

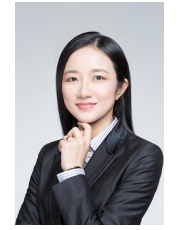

Qian Li received the Bachelor of Science degree from Zhejiang University, Hangzhou, China, in 2003, the Master of Science degree from the Royal Institute of Technology (KTH), Stockholm, Sweden, in 2005, and the Ph.D. degree from the Hong Kong Polytechnic University, Hung Hom, Hong Kong, in 2009. After graduation she was a Visiting Scholar at the University of Washington, Seattle and Postdoctoral Fellow at the Hong Kong Polytechnic University. In 2012 she joined School of Electronic and Computer Engineering (ECE) in Peking University as an Assistant professor. Since 2013 
she is Associate Professor at ECE. Her research interests include nonlinear optics, ultrafast optics and integrated optics. Dr. Li is members of Institute of Electrical and Electronics Engineers (IEEE) and the Optical Society of America (OSA). Currently she is Vice Chair of IEEE ED/SSC Beijing Section (Shenzhen) Chapter and Chair for EDS. From 2015 she is an advisor of OSA Student Chapter in Peking University Shenzhen Graduate School.

Ziyun Jian is Master Student of School of Electronic and Computer Engineering in Peking University.

Wei Lu received his Master degree from School of Electronic and Computer Engineering in Peking University in 2016.

K. Nakkeeran received the B.Eng. degree from the Coimbatore Institute of Technology, Coimbatore, Tamil Nadu, India, in 1993, and the M.Technol. and Ph.D. degrees from Anna University, Chennai, Tamil Nadu, India, in 1995 and 1998, respectively. In 1999, he joined the Institute of Mathematical Sciences, Chennai, where he was a Postdoctoral Fellow for ten months. In 1999, he became a Research Associate with the Department of Physics, University of Burgundy, Dijon, France. In 2002, he became a Postdoctoral Fellow with the Department of Electronic and Information Engineering, The Hong Kong Polytechnic University. In 2005, he joined the School of Engineering, University of Aberdeen, Aberdeen, U.K. Currently he is a Senior Lecturer since 2011. His research interests include solitons, fiber lasers, modeling and simulations of optical devices, long-haul optical fiber communications, and nonlinear science. Dr. Nakkeeran is a member of the Optical Society of America and The Institution of Engineering and Technology (IET).

K. Senthilnathan was born in Kattukkanallur, India, in 1977. He received the M.S. and M.Phil. degrees in physics from the University of Madras, India, and the Ph.D. degree from Anna University, Chennai. He was a Post-Doctoral Fellow with the Department of Electronic and Information Engineering, Hong Kong Polytechnic University, Hong Kong. Currently, he is a Professor in the School of Advanced Sciences, VIT University, Vellore, India. His primary research interests include studying the linear and nonlinear properties of fiber and fiber Bragg gratings and the design of photonic crystal fibers. He received the Gold Medal for the M.Phil. degree. He was sanctioned two research projects. One is under the Young Scientist Scheme by the Department of Science and Technology and another one is under the Council of Scientific and Industrial Research.

P. K. A. Wai (SM'96) received the B.S. (Hons.) degree from the University of Hong Kong in 1981, and the M.S. and Ph.D. degrees from the University of Maryland, College Park, in 1985 and 1988, respectively.

In 1988, he joined Science Applications International Corporation, McLean, VA, where he was a Research Scientist involved with the Tethered Satellite System project. In 1990, he became a Research Associate with the Department of Physics, University of Maryland, College Park, and the Department of Electrical Engineering, University of Maryland, Baltimore County. In 1996, he joined the Department of Electronic and Information Engineering, The Hong Kong Polytechnic University. He became Chair Professor of Optical Communications in 2005. Currently he is the Vice President (Research Development). His research interests include soliton, fiber lasers, modeling and simulations of optical devices, long-haul optical fiber communications, all-optical packet switching, and network theories. He is an active contributor to the field of photonics and optical communications, having authored or coauthored over 300 international refereed publications. Currently he is an associate editor of Journal of Lightwave Technology.

Prof. P. K. A. Wai is the Fellows of the Institute of Electrical and Electronics Engineers (IEEE) and Optical Society of America (OSA). 